\title{
Panxl promotes invasion-metastasis cascade in hepatocellular carcinoma
}

\author{
Guangjun Shi1 ${ }^{,}$, , Chuanliang Liu ${ }^{2,}$, , Yiming Yang $3,{ }^{*}$, Liwei Song 4 , Xueni Liu ${ }^{4}$, Chuanxu Wang1, Zhihai Peng ${ }^{4}$, \\ Hao Li ${ }^{\bowtie}$, Lin Zhong ${ }^{\bowtie}$ \\ 1. Department of Hepatobiliary Surgery, The Affiliated Qingdao Municipal Hospital of Qingdao University, Qingdao, China. \\ 2. Department of General Surgery, The Third People's Hospital of LinYi, Linyi, China. \\ 3. School of Life Science, Shanghai University, Shanghai, China. \\ 4. Department of General Surgery, Shanghai General Hospital, Shanghai Jiao Tong University School of Medicine, Shanghai, China. \\ *These authors contributed equally to this work.
}

$\triangle$ Corresponding authors: Hao Li, Department of General Surgery, Shanghai General Hospital, Shanghai Jiao Tong University School of Medicine, 100 Haining Road, Shanghai 200080, China. Tel: +86-021-63240090-3133, Fax: +86-021-63242903; E-mail: lihao6656@163.com. Lin Zhong, Department of General Surgery, Shanghai General Hospital, Shanghai Jiao Tong University School of Medicine, 100 Haining Road, Shanghai 200080, China. E-mail: zhonglin1@medmail.com.cn.

(1) The author(s). This is an open access article distributed under the terms of the Creative Commons Attribution License (https://creativecommons.org/licenses/by/4.0/). See http://ivyspring.com/terms for full terms and conditions.

Received: 2019.01.09; Accepted: 2019.08.03; Published: 2019.09.07

\begin{abstract}
Background: The molecular function of pannexin 1 ( $P a n x 1)$ in different tumor types has been remained equivocal. Until now, there is no study focused on the function of panxl in hepatocellular carcinoma (HCC). This study aimed to explore the role of Panxl in the invasion and metastasis of HCC.

Methods: The expressions of Panx 1 in 126 cases of $\mathrm{HCC}$ were analyzed by immunohistochemistry (IHC). The effects of Panxl on HCC cell metastasis and invasion were observed by transwell. The expression levels of Panxl and epithelial-mesenchymal transition (EMT) related proteins in HCC cells and tissues were detected by western blot and IHC. The tumor metastatic abilities were compared between Panxl knockout mice and nude mice.

Results: The higher expression of Panxl in HCC was positively correlated with tumor lymph node metastasis, TNM (tumor, node, metastasis) classification and poor prognosis (overall survival, hazard ratio $[H R] 2.769,95 \%$ confidence interval $[95 \% \mathrm{Cl}] 1.528-5.017, P=0.001$; disease-free survival, $\mathrm{HR}=2.344,95 \% \mathrm{Cl} 1.473-3.730, P<0.001$ ). Overexpression of $\mathrm{Panx} 1$ promoted invasion and migration of $\mathrm{HCC}$ cells through modulation of EMT in vitro and in vivo.
\end{abstract}

Conclusions: Our results suggest that the high expression of Panxl is associated with poor HCC prognosis, providing a new clue for effective intervention for HCC metastasis.

Key words: Panx1; hepatocellular carcinoma; metastasis; EMT

\section{Introduction}

Hepatocellular carcinoma (HCC) is one of the most common malignant tumors in the world; it ranked the third leading cause of cancer death [1]. As it is difficult to detect HCC in early stages, only $10 \%-20 \%$ of patients are able to go through radical surgery $[2,3]$. Despite the advanced diagnosis and treatments, the prognosis of patients with HCC remains poor due to the high recurrence and metastasis rate. In addition, the mechanism of HCC is still enigmatic [4-6]. Therefore, the identification of potential biomarkers and novel mechanism of metastasis are of great importance to better understand the occurrence reasons of HCC and provide potential treatments.

Panx1, a channel-forming glycoprotein, was named for its similarities with the invertebrate gap junction protein innexins [7]. Panx1 is a transmembrane domain composed of four alpha 
helices, two extracellular loops and one carboxy terminus [8]. The main function of Panx1 is to form macroporous single-membrane channels that release ATP and metabolites which play a prominent role in the exchange of information between cells [9]. It is widely expressed in most mammalian cells and tissues [10]. The metastasis of primary HCC was considered the main cause of cancer death [11,12]. Recent studies show that Panx1 is involved in the development of many human cancers. Paul et al. reported that Panx1 channel inhibitors reduce ATP release and thereby inhibit breast cancer metastasis [13]. Li et al. found that silencing Panx1 expression can reduce the proliferation of human glioma U87-MG cells [14]. However, in C6 gliomas, Panx1 was reported as a tumor-suppressive factor [15]. Until now, the role of Panx1 in the development of HCC has not been reported yet.

In this study, elevated expression of Panx1 in HCC was positively associated with vascular invasion, tumor metastasis and poor prognosis of patients. This study explained the related mechanism of Panx1 on the HCC invasion and metastasis, which provided a theoretical basis for the diagnosis and prognosis of HCC.

\section{Materials and Methods}

\section{Patient and sample}

126 pairs of HCC and adjacent noncancerous tissue were collected from the Shanghai First People's Hospital between 2009 and 2016. All patients were diagnosed as primary HCC by pathology confirmedly and did not receive any radiotherapy or chemotherapy before surgery. The fresh specimens were processed immediately after surgical removal from the patient's body, then subdivided into three equal portions for histopathologic analysis, quick frozen in liquid nitrogen, or fixated in buffered paraformaldehyde. Staging criteria of HCC were based on the TNM staging which proposed by the Union for International Cancer Control (UICC) and the American Joint Committee on Cancer (AJCC). All patients had provided written informed consent on the use of clinical specimens for biomedical research.

\section{Cell culture}

HCC cell lines (MHCC97H, MHCC97L, MHCC-LM3, HuH7, Hepa1-6), normal liver cells (L02), and HEK 293T were purchased from the cell bank of Chinese Academy of Sciences (Shanghai, China). All cell lines were cultured in Dulbecco's minimal essential medium (DMEM, Invitrogen, NY, USA) containing $10 \%$ fetal bovine serum (FBS, Invitrogen) and $1 \%$ penicillin/streptomycin antibiotics (Invitrogen) and were incubated in an incubator with $5 \% \mathrm{CO} 2$ at $37^{\circ} \mathrm{C}$.

\section{Experimental animals}

C57BL/6J mice and nude mice were purchased from Shanghai Laboratory Animal Center (SLAC). Male C57BL/6 mice (specific pathogen-free (SPF)-grade) and Panx1 knockout mice (aged 6-8 weeks) were bred at the animal facility of Institute Pasteur of Shanghai under pathogen-free conditions. The nude mice were housed with free access to standard laboratory diet and water. All animal experiments in this section are approved by the Laboratory Animal Ethics Committee of the Chinese Academy of Sciences.

\section{Plasmids and transfection}

Panx1 overexpression plasmid and Panx1 control were purchased from Shanghai Jima Pharmaceutical Technology Co. Ltd. A three-plasmid-based virus packaging cell system was established, consisting of pCMV-dR8.91, pCMV-VSVG, Panx1-OE (over-expressed) / Panx1-OE-control. The three plasmid system co-transfected into HEK 293T cells, and the virus-infected 97L and LM3 cells were collected. Puromycin $2 \mu \mathrm{L}$ was screened (concentration 10 $\mathrm{mg} / \mathrm{mL}$ ) after $48 \mathrm{~h}$. The stably expressing Panx1 of cells was verified by western blotting.

\section{Immunohistochemistry (IHC)}

The paraffin sections were incubated with primary antibody. Each slide was designated with a score for density and intensity. The staining score and the intensity score of the positive cells on each slide were calculated using the semi-quantitative scoring method. Specifically, staining scores were assigned according to the percentage of positive tumor cells, as follows: 1 (up to $25 \%$ positive cells), $2(25 \%-50 \%$ positive cells), 3 (50\%-75\% of positive cells), and 4 (more than $75 \%$ positive cells). The intensity scores were set between 0 and 3 , which are designated as follows: 0 , no staining; 1 , weak staining; 2 , moderate staining; and 3, strong staining. The final results ranging from 0 to 12 were obtained with the multiple of the staining score and the intensity score. A total score of 0-6 indicates low expression, whereas a total score of 7-12 indicates high expression.

\section{Cell migration and invasion assays}

The invasive abilities of 97L and LM3 cells were detected by trans-well culture system. For invasion, the trans-well membrane of the upper chamber coated with Matrigel (BD Biosciences Discovery Labware, Woburn, MA, USA) was used. The chambers were rehydrated with serum-free medium for $2 \mathrm{~h}$ at $37^{\circ} \mathrm{C}$ in an incubator. The top chambers were subsequently 
hydrated with $200 \mathrm{ul}$ of cell suspension (containing 1 $\times 105$ cells). The bottom chambers were added with $500 \mathrm{ul}$ of medium containing $10 \%$ FBS as a chemoattractant. After culturing for 24 hours at $37^{\circ} \mathrm{C}$, the transmembrane cells on the lower surface of the membrane were fixed with formaldehyde at room temperature for $5 \mathrm{~min}$, stained with crystal violet for $20 \mathrm{~min}$, washed three times with clean water, and counted under a microscope.

\section{Western blot analysis}

Western blot analysis was implemented on standard procedures [16]. The following antibodies were used in the experiments: anti-GAPDH antibody (Abcam, Cambridge, UK), $\beta$-actin antibody (Abcam, Cambridge, UK), anti-Panx1 antibody (Abcam, Cambridge, UK), anti-E-cadherin antibody (Cell Signaling Technology, BO, USA), anti-Vimentin antibody (Cell Signaling Technology, BO, USA), anti-Snail antibody (Cell Signaling Technology, BO, USA), anti-MMP2 antibody (Cell Signaling Technology, BO, USA), total AKT antibody (Cell Signaling Technology, BO, USA), and p-AKT (Ser 473) (Cell Signaling Technology, BO, USA). Cell and tissue sample lysates were electrophoresed using 10\% sodium dodecyl sulfate-polyacrylamide gels and then transferred onto polyvinylidene difluoride membranes (Millipore, USA). The membranes were incubated with primary antibodies for $1-2 \mathrm{~h}$ at room temperature overnight at $4{ }^{\circ} \mathrm{C}$ and then incubated with the related secondary antibody for $1 \mathrm{~h}$ at room temperature. Protein detection was performed using an enhanced chemiluminescence kit (Ab Frontier), and the blots were exposed to X-ray films. Band intensities were quantified using ImageJ software (version 1.43; National Institutes of Health, Bethesda, MD, USA) with GAPDH as the loading control.

\section{In vivo detection of tumor invasion and metastasis}

Ten nude mice were randomly divided into two groups. LM3 cells $\left(1 \times 10^{6}\right.$ cells/mice $)$ stably transfected with retrovirus-Panx1 or retrovirus-control vector were injected by tail vein injection. The mice were sacrificed by dislocation before observing the lung metastasis two weeks later. The tumor metastases were compared between Panx1 knockout mice and wild-type mice. Hepa1-6 cells were planted in the armpit of mice and the tumor size was measured regularly.

\section{Statistical Analysis}

Calculations were performed with GraphPad InStat, Version 5.0 (GraphPad Prism Software, San Diego, CA, USA). Student's two-tailed t-test was utilized for all data analysis and values were expressed as the mean \pm standard error of the mean acquired from at least two independent experiments. $P<0.05$ was considered to indicate a statistically significant difference.

\section{Results}

\section{High Panxl expression was an independent prognostic factor for patients with resectable HCC}

To determine the clinical significance of Panx1, we performed survival analysis in 126 patients with HCC. Baseline characteristics of the patients are listed in Supplementary table 1. Patients were divided into two groups based on their Panx1 expression levels. Panx1 expression levels was positively correlated with microvascular involvement $(P=0.008)$ and tumor lymph node metastasis stages $(P=0.020)$ in patients with HCC. Patients with high Panx1 expression in tumors displayed a more advanced disease state (stages 3 and 4) than patients with low expression of Panx1 in tumors $(P=0.022)$. Kaplan-Meier analysis showed that patients with high Panx1 expression in HCC tissues displayed shorter overall survival (OS) and disease-free survival (DFS) than those in patients with low Panx1 expression $(P=0.009$ and $P=0.004$, respectively) (Figure 1A). A receiver operating characteristic (ROC) curve analysis were performed to evaluate the prognosis value of Panx1 and the results showed that the area under the curve (AUC) for Panx1 expression associated with OS was 0.675 and DFS 0.701, respectively (Figure 1B). The Cox regression analysis displayed that Panx1 expression (HR=2.344, $\quad P<0.001)$, Microvascular involvement $(\mathrm{HR}=3.426, P=0.005)$, and TNM stage $(\mathrm{HR}=4.029$, $P=0.007)$ were significantly associated with DFS in HCC patients (Table 1). Panx1 expression $(\mathrm{HR}=2.769$, $P=0.001)$, and TNM stage $(\mathrm{HR}=10.233, P=0.032)$ were significantly associated with OS in HCC patients (Table 2). These findings suggest that Panx1 expression was considered independent prognostic factors for HCC.

\section{Panxl promoted the invasion and metastasis of HCC cells in vitro and in vivo}

LM3 and 97L cells were transfected with the retrovirus-Panx1 or retrovirus-control vector. Trans-well results showed that Panx1 overexpression significantly promoted the invasion and metastasis of LM3 and 97L cells (Figure 2A, B). Several EMT-related genes, the expressions of Snai1, Vimentin and MMP2 were up-regulated whereas E-cadherin was down-regulated by overexpression in HCC cells (Figure 2C). In subcutaneous tumor in nude mice, 
IHC showed that the Vimentin, Snail, and MMP2 positive staining were significantly more frequent in tumors of Panx1-transfected HCC cells than that in control cells, while E-cadherin protein expression was reduced in the Panx1 overexpression group, indicating that overexpression of Panx1 was more helpful to the occurrence of EMT (Figure 2D). To testify whether Panx1 overexpression contributes to tumor metastasis in vivo, we injected LM3-stable cells (overexpression and control) into the tail vein of nude mice. These results indicated that Panx1 overexpression significantly promoted lung metastasis in nude mice (Figure 2E).

\section{Panxl promoted EMT of HCC were dependent on AKT signaling}

To search for the potential link between Panx1 and the EMT signaling, we examined the molecular

$\mathbf{A}$
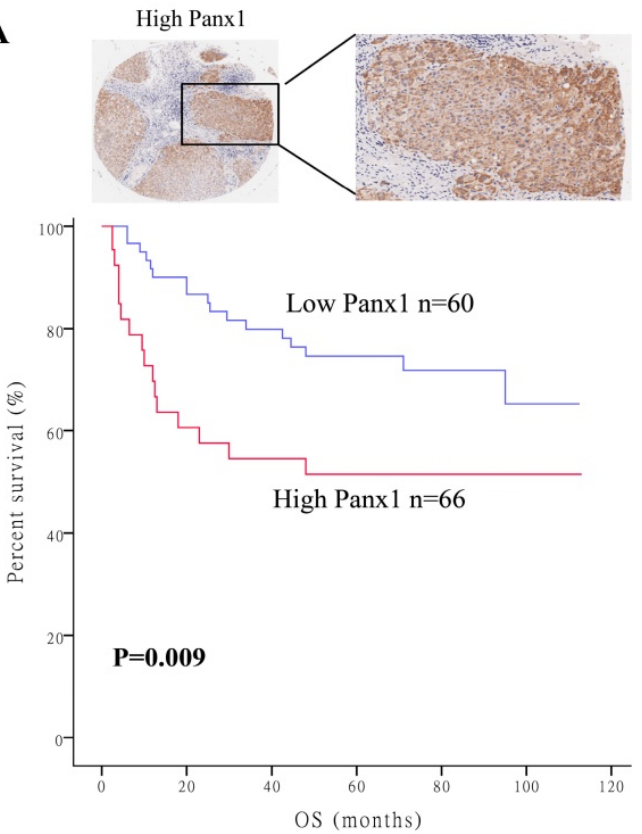

B

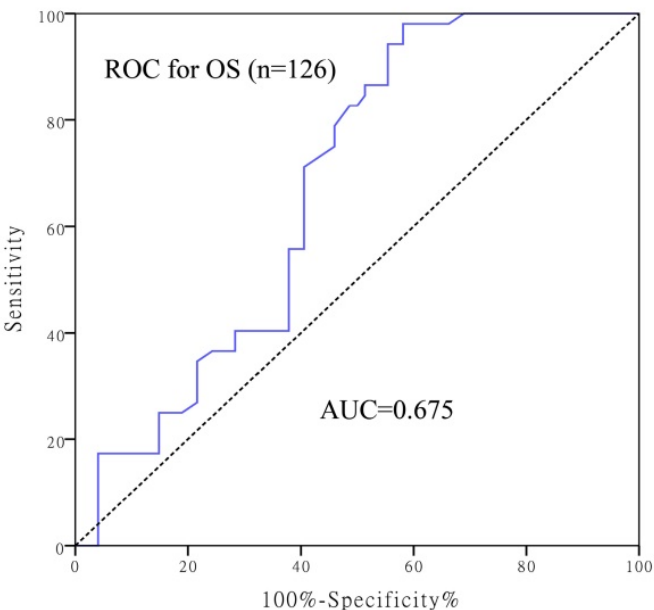

alterations in AKT signaling pathways that were important in EMT induction. The results showed that Panx1 promoted EMT of HCC via phosphorylated AKT in both LM3 and 97L cells (Figure 3).

\section{Panxl knockout inhibited metastasis in vivo}

The hepa1-6 cells were injected in the axilla of Panx1 knockout mice and wild-type (WT) mice respectively. The tumor volume in Panx1 knockout mice was significantly smaller than those in WT mice (Figure 4A). The number of lung metastasis tumor nodules in Panx1 knockout mice was significantly reduced than that in WT mice (Figure 4B). These data indicated that Panx1 knockout inhibited its metastasis.
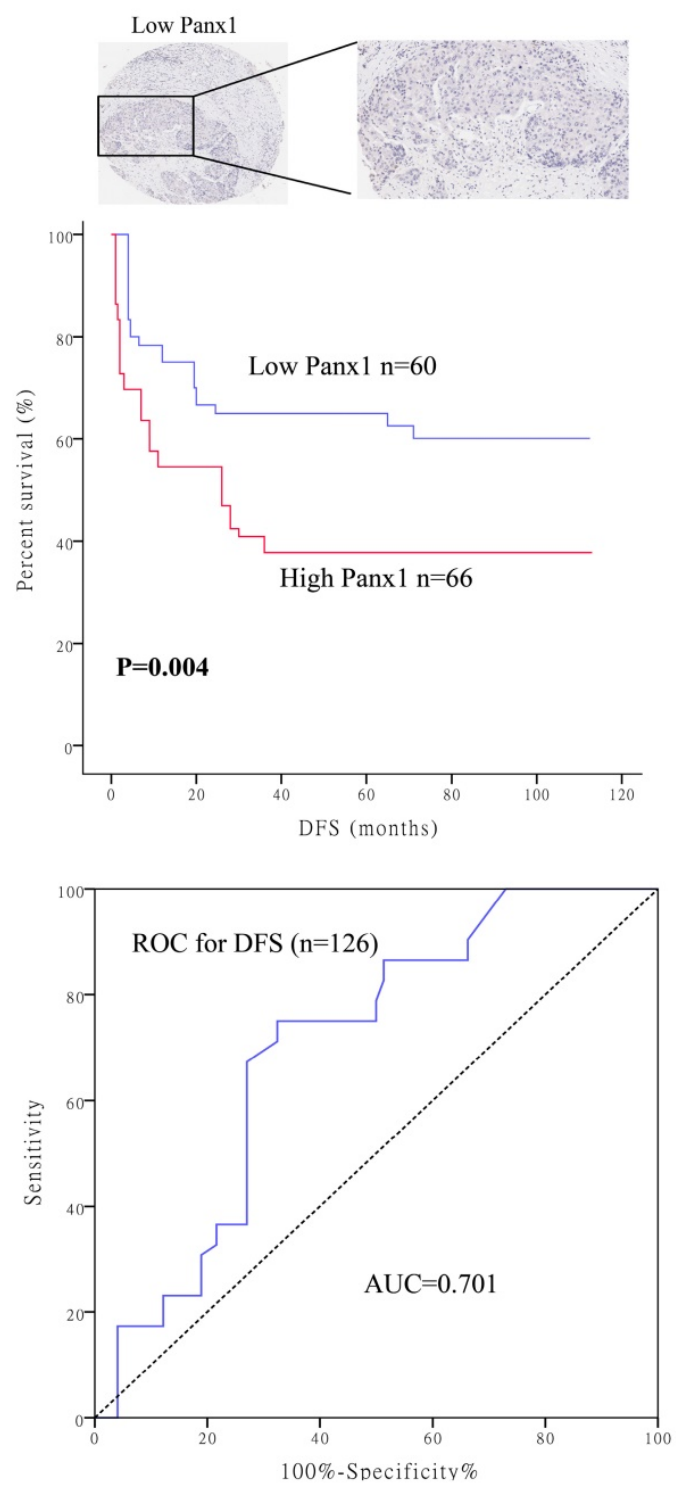

Figure 1. High Panxl expression was an independent prognostic factor for patients with resectable HCC. (A) Kaplan-Meier showed that patients with high Panxl expression in tumors displayed short overall survival $(P=0.009)$ and disease-free survival $(P=0.004)$ compared with those showing low $P a n x 1$ expression. $(B)$ ROC curve of $P a n x 1$ for OS (AUC $=0.675)$ and DFS $(A \cup C=0.701)$ in PDAC patients. 
Table 1. Univariate and multivariate analysis of factors associated with DFS of HCC patients

\begin{tabular}{lll}
\hline Variables & Hazard ratio $(95 \% \mathrm{CI})$ & $P$ value \\
\hline Univariate analysis & & \\
Panx1 (low, moderate vs high) & $1.824(1.187-2.801)$ & 0.006 \\
Gender (male vs female) & $1.446(0.342-6.112)$ & 0.616 \\
Age $(>50$ vs $\leq 50)$ & $1.188(0.534-2.647)$ & 0.673 \\
HBV (positive vs negative) & $0.383(0.051-2.854)$ & 0.349 \\
Tumor size ( $>5 \mathrm{~cm}$ vs $\leq 5 \mathrm{~cm})$ & $3.082(1.423-6.674)$ & 0.004 \\
Liver cirrhosis (yes vs no) & $0.544(0.219-1.351)$ & 0.189 \\
Microvascular involvement (positive vs & $4.803(2.223-10.380)$ & $<0.001$ \\
negative) & & \\
\hline
\end{tabular}

A
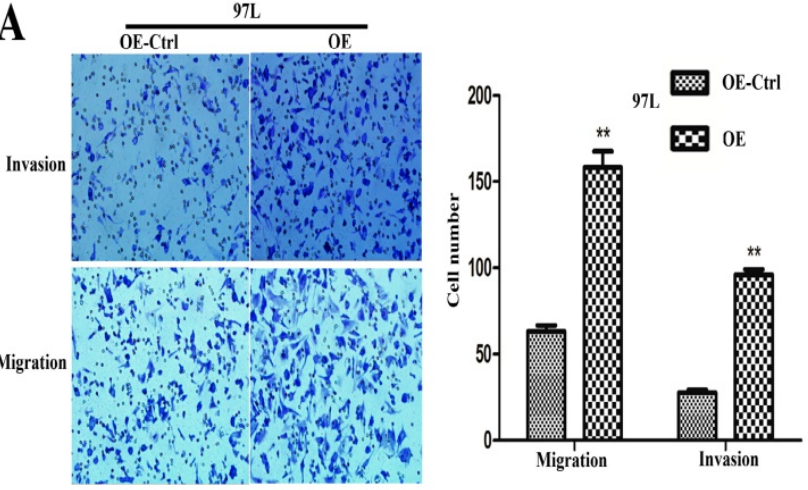

C

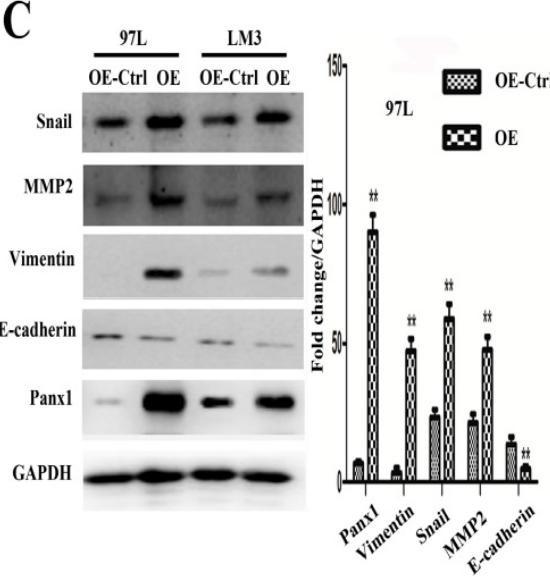

E

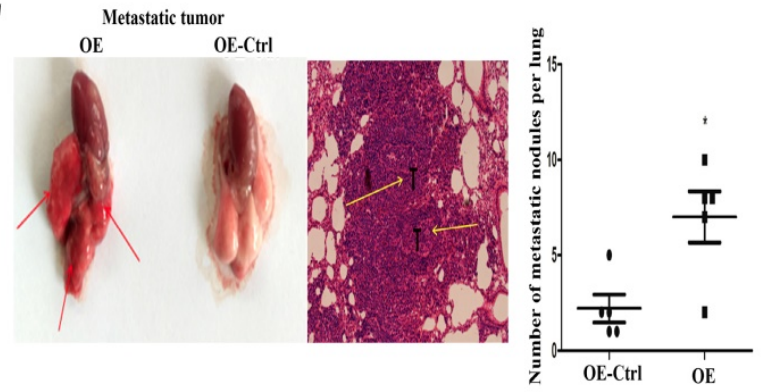

Variables

Hazard ratio $(95 \% \mathrm{CI})$

Differentiation (Poorly vs well+moderately)

TNM stage (III vs I-II)

(1.088-6.805)

$5.144(1.938-13.655)$

$1.494(0.563-4.729)$

$0.893(0.360-2.212)$

$2.344(1.473-3.730)$

4.029 (1.476-10.999)

Multivariate analysis

Panx1 (low, moderate vs high)

TNM stage (III vs I-II)

$3.426(1.451-8.090)$ negative)

$\mathrm{P}<0.05$ was considered statistically significant

\section{value}

0.001

0.102

0.806

$<0.001$

0.007

0.005
B

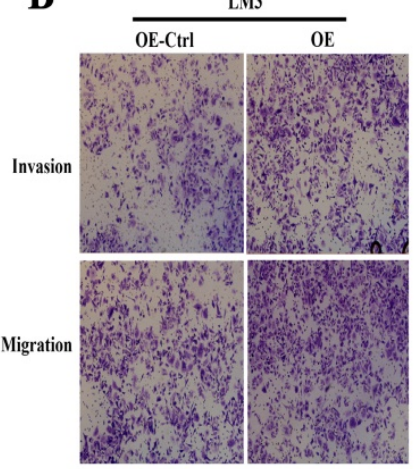

D
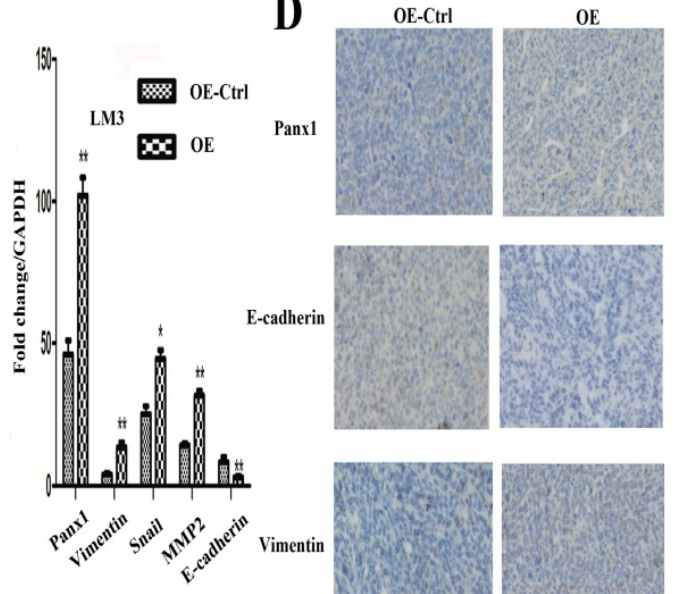
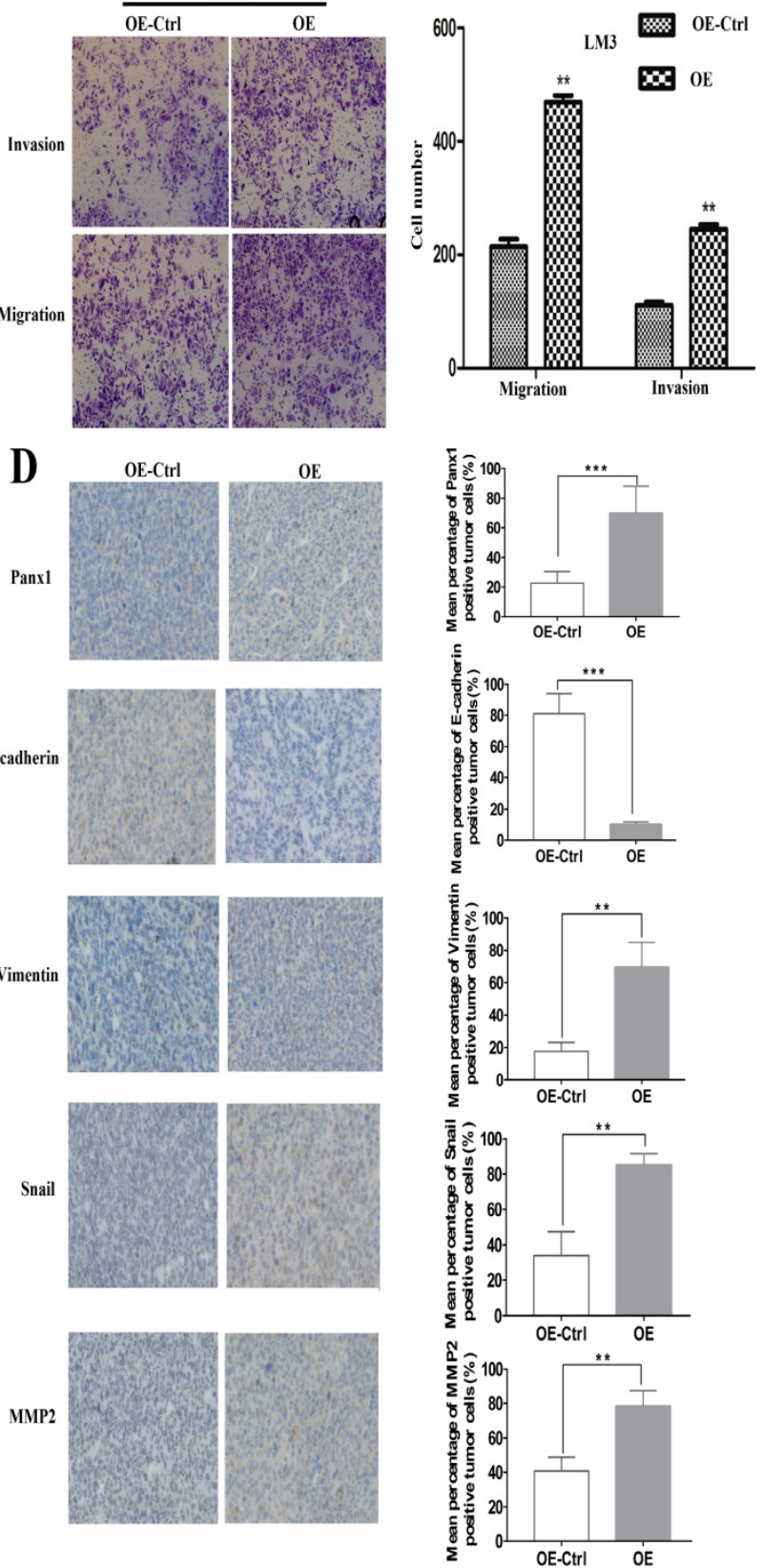

Figure 2. Overexpression of Panxl promoted cell invasion and metastasis in vitro and in nude mice. (A, B) Transwell invasion and metastasis assay showed that overexpression of Panxl enhanced the invasion and metastasis ability of HCC cell lines ( $97 \mathrm{~L}$ cells and LM3 cells). (C) Western blot detected the expression of Panxl, E-cadherin, Vimentin, MMP-2, and Snail in 97L and LM3 cells transfected with OE or OE-Ctrl. (D) IHC showed that the expression of Vimentin, Snail, and MMP2 protein increased in tumors formed from the Panxl-transfected HCC cells than that in control cells, while E-cadherin protein expression was reduced in the Panxl overexpression group. (E) Overexpression of Panxl enhanced lung metastasis of HCC in nude mice. 
A
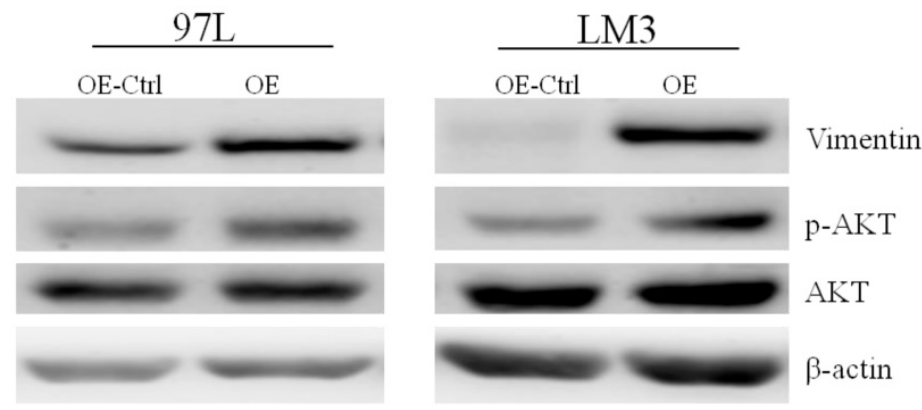

B
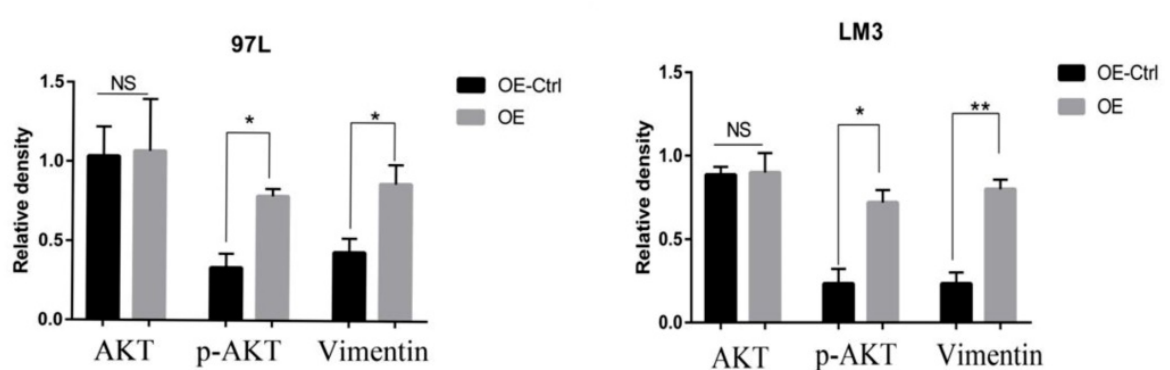

Figure 3. Panxl promoted EMT of HCC cells by AKT phosphorylation. (A) Western blot detected the expression of AKT, p-AKT, and Vimentin in 97L and LM3 cells transfected with OE or OE-Ctrl. (B) The average relative density of AKT, p-AKT, and Vimentin in 97L and LM3 cells.

A
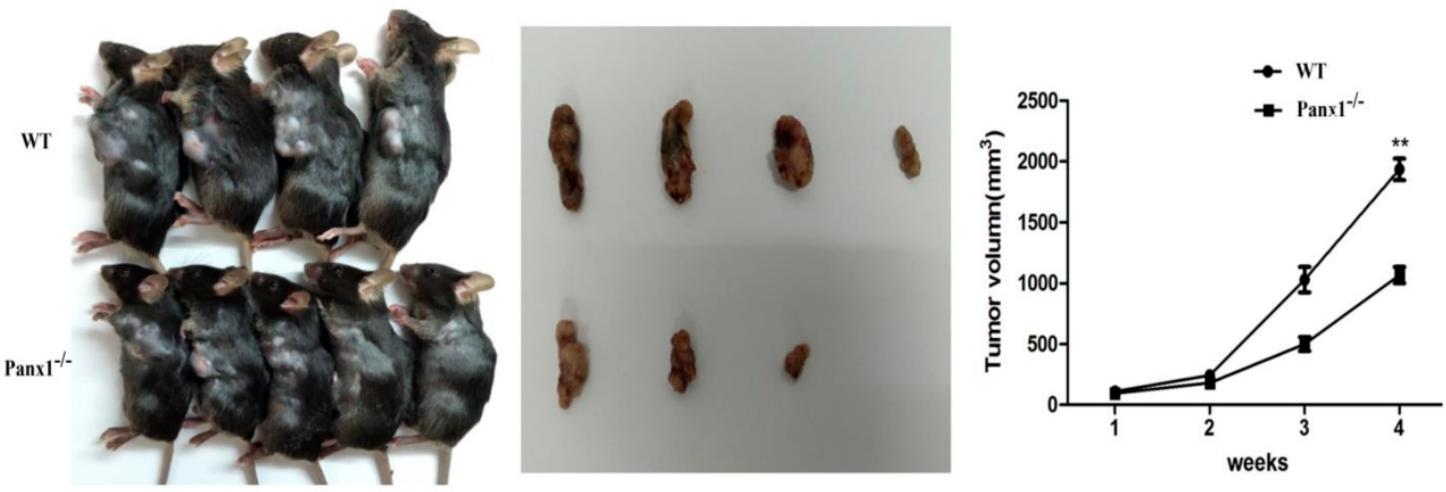

B
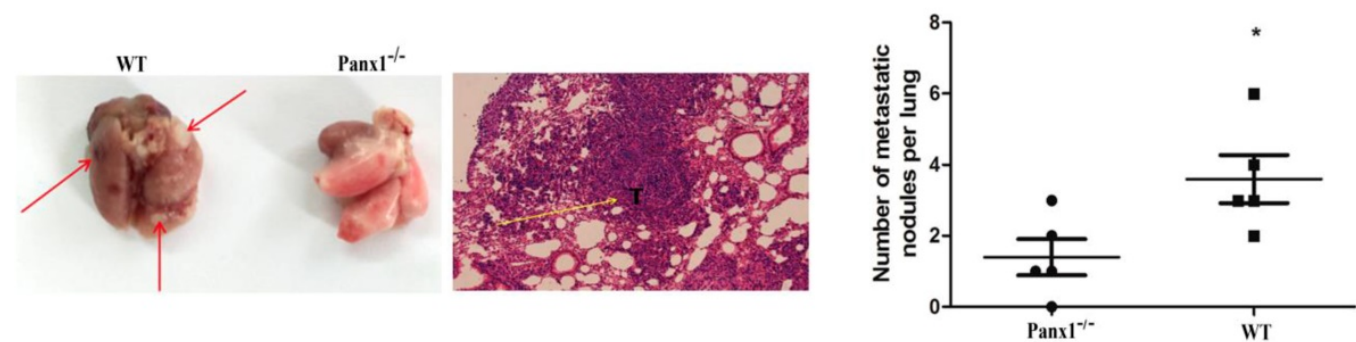

Figure 4. Deleted of Panxl suppressed tumor cells lung metastasis in Panxl knockout mice. (A) Panxl knockout mice suppressed HCC growth. (B) Panxl knockout mice suppressed lung metastasis of HCC.

Table 2. Univariate and multivariate analysis of factors associated with OS of HCC patients

\begin{tabular}{lll}
\hline Variables & Hazard ratio $(95 \% \mathrm{CI})$ & $P$ value \\
\hline Univariate analysis & & \\
Panx1 (low, moderate vs high) & $3.064(1.693-5.544)$ & $<0.001$ \\
Gender (male vs female) & $0.867(0.199-3.771)$ & 0.849 \\
Age $(>50$ vs $\leq 50)$ & $1.118(0.419-2.979)$ & 0.824 \\
HBV (positive vs negative) & $0.764(0.625-1.201)$ & 0.232 \\
Tumor size (>5cm vs $\leq 5 \mathrm{~cm})$ & $2.265(0.892-5.750)$ & 0.085 \\
Liver cirrhosis (yes vs no) & $0.333(0.124-0.892)$ & 0.029 \\
\hline
\end{tabular}

\begin{tabular}{lll}
\hline Variables & Hazard ratio (95\% CI) & $P$ value \\
\hline $\begin{array}{l}\text { Microvascular involvement (positive vs } \\
\text { negative) }\end{array}$ & $7.261(2.782-18.951)$ & $<0.001$ \\
$\begin{array}{l}\text { Differentiation (Poorly vs } \\
\text { well+moderately) }\end{array}$ & $1.663(0.481-5.756)$ & 0.422 \\
TNM stage (III vs I-II) & $18.908(2.509-142.487)$ & 0.004 \\
Lymph node metastasis (yes vs no) & $2.854(1.051-7.972)$ & 0.047 \\
AFP (>20ng/ml vs $\leq 20 \mathrm{ng} / \mathrm{m})$ & $1.508(0.566-4.019)$ & 0.412 \\
$\begin{array}{l}\text { Multivariate analysis } \\
\text { Panx1 (low, moderate vs high) }\end{array}$ & $2.769(1.528-5.017)$ & 0.001 \\
TNM stage (III vs I-II) & $10.233(1.226-85.410)$ & 0.032 \\
\hline$P<0.05$ was considered statistically significant &
\end{tabular}




\section{Discussion}

Although the clinical diagnosis and surgical treatment of HCC have made great progress, the mortality rate of HCC is still amongst the top three highest. The majority of patients with HCC have undergone intrahepatic or even distal metastasis in clinical treatments. Therefore, the development of new hepatocellular carcinoma diagnosis and prognostic factors is of urgent significance. Previous studies have revealed that Panx1 channels are involved in the regulation of cellular functions mainly through the mediation of ATP release and paracrine signaling [17-19]. However, this study mainly focused on the relationship between the expression level of Panx1 and the clinical indications in HCC patients as well as the role of Panx1 in HCC metastasis.

The expression of Panx1 was positively correlated with tumor TNM staging and venous invasion, negatively correlated with overall survival. At the same time, Panx1 expression levels and venous invasion could be used as an independent prognostic factor for the patients with HCC. Consistent with this, Stewart et al. reported that high expression levels of Panx1 are associated with poor OS of breast cancer patients [20]. This provides a new direction for us to find a novel indicator of HCC prognosis.

As we know metastasis is the main factor for HCC patients with a poor prognosis, and we speculated that Panx1 may have an related impact on the biological function of hepatocellular carcinoma cells. We established a Panx1 over-expressing hepatocellular carcinoma cell line and found that Panx1 overexpression promotes cell migration and invasion. Consistent with this, Silvia reported that the loss of Panx1 expression inhibits cell proliferation and migration on melanoma [21]. In Lai's report, on the contrary, they found that overexpression of Panx1 in gliomas suppresses cell malignancy [15]. The above researches on Panx1 in different tumors indicate that Panx1 plays a complex role in the development of cancer, resulting in different data which might relate to the genetic background of tumor cells, cell culture conditions or analytical methods of the researchers, which needs more researchers to explore and discuss. In this study, Panx1 was found to promote the transmembrane transformation of hepatocellular carcinoma cells by promoting the expression of Snai1, an EMT-related transcription factor. The expression of E-cadherin protein was down-regulated, the expression of vimentin and snail protein was up-regulated, and the expression of MMP2 protein was up-regulated, suggesting that Panx1 overexpression may promote cell migration and invasion. Subsequently, we used trans-well to detect the metastatic potential of the cells, comparing it with the control group, the ability of migration and invasion of the hepatocarcinoma cells with Panx1 overexpression group was significantly increased.

This showed that Panx1 can regulate cell migration and invasion by promoting epithelial-mesenchymal transition. However, the main focus of our follow-up study is to find out how Panx1 affects and regulates EMT-related gene expression. To further clarify the role of Panx1 in tumorigenesis, we established a tumorigenic model of subcutaneous nude mice with HCC cells and found that Panx1 promoted the tumor volume significantly. Meantime, we also planted hepa1- 6 cells in the axilla of Panx1 knockout mice and obtained the same results, the lung metastasis of mice in the Panx1 knockout group was significantly lower than the wild-type. This showed that Panx1 can promote cell migration, which is consistent with previous experiments in vitro.

This present study investigated the clinical significance of Panx1 in HCC patients, but it still has some limitations. First, although high expression of Panx1 can predict the poor prognosis in HCC, it still lacks of registered clinical trial. Second, although we confirmed Panx1 promotes HCC invasion and metastasis in vitro and in vivo (included nude mice and Panx1 knockout mice), as well as EMT-related expression, we did not provide the detailed mechanism about how Panx1 promotes EMT-related expression.

The above studies showed that Panx1 is involved in the development of HCC and promote cell migration. In summary, this study firstly proved the role of Panx1 in the development of HCC, however, the mechanism remains to be determined.

\section{Supplementary Material}

Supplementary table.

http://www.jcancer.org/v10p5681s1.pdf

\section{Acknowledgements}

This work was partially supported by grants from the National Natural Science Foundation of China (81702341, 81170447) and the Medical Engineering Crossing Project Grant Funded by Shanghai Jiaotong University (YG2016QN24).

\section{Competing Interests}

The authors have declared that no competing interest exists.

\section{References}

[1] Forner A, Llovet JM, Bruix J. Hepatocellular carcinoma. Lancet. 2012;379:1245-55.

[2] Kudo M. Systemic Therapy for Hepatocellular Carcinoma: Latest Advances. Cancers (Basel). 2018;10: E412. 
[3] Clark HP, Carson WF, Kavanagh PV, Ho CP, Shen P, et al. Staging and current treatment of hepatocellular carcinoma. Radiographics. 2005;1:S3-23.

[4] Niu ZS, Niu XJ, Wang WH. Genetic alterations in hepatocellular carcinoma: An update. World J Gastroenterol. 2016;22:9069-9095.

[5] Dhir M, Lyden ER, Smith LM, Are C. Comparison of outcomes of transplantation and resection in patients with early hepatocellular carcinoma: a meta-analysis. HPB (Oxford). 2012;14:635-45.

[6] Wong R, Frenette C. Updates in the management of hepatocellular carcinoma. Gastroenterol Hepatol (N Y). 2011;7:16-24.

[7] Baranova A, Ivanov D, Petrash N, Pestova A, Skoblov M, Kelmanson I, et al. The mammalian pannexin family is homologous to the invertebrate innexin gap junction proteins. Genomics. 2004;83:706-16.

[8] Bruzzone R, Hormuzdi SG, Barbe MT, Herb A, Monyer H. Pannexins, a family of gap junction proteins expressed in brain. Proc Natl Acad Sci U S A. 2003;100:13644-9.

[9] Penuela S, Bhalla R, Gong XQ, Cowan KN, Celetti SJ, Cowan BJ, et al. Pannexin 1 and pannexin 3 are glycoproteins that exhibit many distinct characteristics from the connexin family of gap junction proteins. J Cell Sci. 2007;120:3772-83.

[10] Celetti SJ, Cowan KN, Penuela S, Shao Q, Churko J, Laird DW. Implications of pannexin 1 and pannexin 3 for keratinocyte differentiation. J Cell Sci. 2010;123:1363-72.

[11] Hurst DR, Welch DR. Metastasis suppressor genes at the interface between the environment and tumor cell growth. Int Rev Cell Mol Biol. 2011;286:107-80.

[12] Eccles SA1, Welch DR. Metastasis: recent discoveries and novel treatment strategies. Lancet. 2007;369:1742-57.

[13] Furlow PW, Zhang S, Soong TD, Halberg N, Goodarzi H, Mangrum C, et al. Mechanosensitive pannexin- 1 channels mediate microvascular metastatic cell survival. Nat Cell Biol. 2015;17:943-52.

[14] Wei L, Yang X, Shi X, Chen Y. Pannexin 1 silencing inhibits the proliferation of U87 MG cells. Mol Med Rep. 2015;11:3487-92.

[15] Lai CP, Bechberger JF, Thompson RJ, MacVicar BA, Bruzzone R, Naus CC. Tumor-suppressive effects of pannexin 1 in C6 glioma cells. Cancer Res. 2007;67:1545-54.

[16] Li H, Zhou ZQ, Yang ZR, Tong DN, Guan J, Shi BJ, et al. MicroRNA-191 acts as a tumor promoter by modulating the TET1-p53 pathway in intrahepatic cholangiocarcinoma. Hepatology. 2017;66:136-151.

[17] Michalski K, Kawate T. Carbenoxolone inhibits Pannexin1 channels through interactions in the first extracellular loop. J Gen Physiol. 2016;147:165-74.

[18] Schalper KA, Carvajal-Hausdorf D, Oyarzo MP. Possible role of hemichannels in cancer. Front Physiol. 2014:5·237.

[19] Bond SR, Naus CC. The pannexins: past and present. Front Physiol. 2014;5:58

[20] Stewart MK, Plante I, Penuela S, Laird DW. Loss of Panx1 Impairs Mammary Gland Development at Lactation: Implications for BreastTumorigenesis. PLoS One. 2016;11:e0154162.

[21] Penuela S, Gyenis L, Ablack A, Churko JM, Berger AC, Litchfield DW, et al. Loss of pannexin 1 attenuates melanoma progression by reversion to a melanocytic phenotype. J Biol Chem. 2012;287:29184-93. 